\title{
Notes on petroleum industry standard geological probability assessments
}

\author{
Imre Szilágyi \\ Exploration Geologist and Petroleum Economist \\ Independent Expert, Budapest
}

\begin{abstract}
This paper discusses the assessment methodology of geologic probabilities of success of drillable prospects determined by petroleum exploration geologists. The commonly accepted industry standard assessment methods suggest the probability evaluation of key components of oil and gas accumulation: source rock, reservoir, seal rock and trap, and migration and timing. On the assumption that the risked events resulting in the assembly of the key components are geologically and eventalgebraically independent, the geological probability is computed as the product of the probabilities of each. Without challenging the overall correctness of the approach, this study argues that the presumed independency does not always apply. For these cases, probability evaluation of the actually and truly independent geologic and hydrodynamic processes is advised.
\end{abstract}

Key words: petroleum exploration drilling projects, geological probability components, probability of independent events

\section{Introduction}

Geological probability $(\mathrm{Pg})$ describes the chance that an exploratory well targeting a potential subsurface oil and gas-bearing geologic structure, commonly referred to as a prospect, will prove recoverable quantities of petroleum. In fact the $\mathrm{Pg}$ is a subjective expert opinion of geoscientists responsible for the development and evaluation of geologic models of potential petroleum accumulations.

Assessing the Pg is probably the most important management process in the petroleum exploration industry. Its relevance lies in the role it plays in the determination of the economic value of an exploratory drilling project. The

Address: I. Szilágyi: H-2040 Budaörs, Kertész utca 22/1, Hungary, e-mail: im.szilagyi@hotmail.com Received: January 24, 2013, accepted: March 7, 2013 
economic value is referred to as the Expected Net Present Value (ENPV) and is based on the principles of Decision Analyses (Howard 1966). It is computed as follows (modified after Newendorp 1975 and Rose 1992):

$$
E N P V=P g \times\left(N P V-C_{E X P}\right)-(1-P g) \times C_{D H}
$$

where NPV (Net Present Value) is the economic value of the future appraisal and field development project (what may follow the discovery), $C_{E X P}$ is the (discounted) cost of the successful exploration, $\mathrm{C}_{\mathrm{DH}}$ is the (discounted) cost of the unsuccessful exploration (or "dry-hole cost"), and Pg is the geological probability of the discovery. (Note that the formula applies if the exploratory drilling targets a single prospect. In multi-prospect target cases Pg is replaced by the Probability of Geologic Success - PoGS - which is derived from the geologic probabilities of the prospects to drill.)

The ENPV - like other expected values under the principles of Decision Analyses - serves as the primary information for the management of the profitability of the exploration project and is thus the most important project ranking factor. Since ENPV is basically influenced by $\mathrm{Pg}$ the appropriate assessment of the parameter seems essential.

\section{Industry standard assessments}

To date the most comprehensive discussion of Pg is given by Rose (2001). The study suggests a five-component system where the geologic chance factors subject to probability analysis are the existence of hydrocarbon source rocks, the occurrence of the fluids' migration from source rock to trap, the presence of a charged and effectively producible reservoir, the existence of closure, and finally containment ensured by the presence of seal rocks blocking the upward migration of fluids from the reservoir. A four-component analysis based on a slightly different approach is presented by Otis and Schneidermann (1997). Beside source rock, reservoir and trap (closure) the authors merge the migration and containment components into a single one named play dynamics. To the best of my knowledge today's exploration ventures use one of the above systems for geological probability assessment.

Both methods agree that the evaluators may subjectively consider geologic evidence (which is inherited geologic and geophysical data and information) to numerically assess the probability of each of the above components. According to the commonly accepted quantification guidelines the probability of 1.0 should be given if the evidence unequivocally proves the occurrence of a component; the probability is 0 if the evidence clearly disproves it (which means that that there is no chance to find hydrocarbons); while it should be scored at around 0.5 if there is no evidence for a given component, or the evidence is unreliable, or the positive and negative evidence is in balance. Values given in the range of 0 to 0.5 
and 0.5 to 1 may reflect the evaluators' degree of non-confidence or confidence, respectively, in the occurrence of the actual component. Recommendations for further refinement of probability scoring are not detailed in this study but are available in the articles cited.

Another relevant similarity in both approaches is that the probability components suggest considering certain sub-factors. For instance in Rose (2001), for the source rock component it is advised to evaluate the quantity (thickness, extent, organic richness), the generated hydrocarbon type (oil, gas, mixed) and the thermal maturity in parallel. Concerning the sub-factors the authors emphasize an important restriction: they must be the result of - at least partly dependent geologic events. The event-algebraic dependency means that the probability of any of the sub-factors influences the probability of the others (in the example above: if a higher probability is given for a source rock quantity, then a higher score should be given for thermal maturity as well). Based on the presumption of dependency, Rose (2001) concludes that the overall probability of a component should be equal to the lowest probability score of any of the subfactors (thus if the probabilities of source rock quantity, fluid type and maturity are $0.8,0.6$ and 0.9 , respectively, then the overall source rock probability is 0.6$)$. This assumption, for which no mathematical reasoning is given, is referred to in Rose (2001) as a "weak link" approach. The discussion whether sophisticated mathematical modeling - e.g. using the Bayesian approach - are more suitable for the probability assessment of algebraically independent events is beyond the scope of this study.

Once the assessment of the probabilities of all the components is completed the prospect's overall geological probability $(\mathrm{Pg})$ is computed as follows:

$$
P g=\prod_{i} P(C)_{i}
$$

where $C$ is a geological probability component, $i$ is the number of the components.

Applying multiplication implies that the components are independent, both geologically and event-algebraically. The consequence of the presumed independency is that the actual probability of any of the components does not influence the probability of any of the others. Implicitly it means that the corresponding events should also be independent of one another. The other very important implication is that the number of the independent events should be equal to the components to be multiplied.

Undoubtedly the geologic models of the most of the prospective hydrocarbon accumulations meet the above assessment criteria and therefore the commonly applied "traditional" methods discussed above can be used for the majority of the cases without any concern. There are, however, some exceptions requiring unique treatment. 


\section{Exceptions}

Examples for exceptions from the generally applicable models are discussed below in two categories. The first category represents cases where one "traditional" geological probability component is in fact the result of two independent events. The other study set includes those examples where two separate "traditional" factors are the results of a single event.

\section{One component - Two independent events}

We can find many examples for structural traps where the presumed closure is the result of at least two separate tectonic events. The simplest case might be a nearly isometric anticline generated by two compressive tectonic events which are separated in geologic time (f.i. N-S folding is assumed to have taken place in the Late Cretaceous, and a perpendicular W-E folding during the Early Miocene). The probabilities of the favorable outcomes of the two events must differ because the probability given for any of the two outcomes does not influence what probability should be assessed for the other. In this example we can assess, merely by considering a variety of evidence, the probabilities of the two outcomes independently, and the probability of the final favorable outcome (i.e. we have an anticlinal trap) is given by the multiplication of the two.

Another fine example might be the separation of source rock deposition and maturation. Let us suppose that the rock formation was deposited in the Devonian but the thermal subsidence resulting in the maturation took place in the Eocene. Needless to say that the evidence for the two favorable outcomes will be different. Should we assess a higher probability for favorable deposition, this will not influence the probability given for favorable maturation. This means that the probabilities of favorable deposition and maturation are independent. Of course the probability of finally having a good source rock will increase but this is due to the higher probability of one of the multiplication factors - in our example it is deposition.

\section{Two components - One event}

The example in this category is when the source rock and the seal rock are in fact the same formation (f.i. Eocene marl). The probability evaluation should address the occurrence of the formation "as is". Although we may give a higher or lower probability to the attribute which makes the marl a seal (namely its impermeability) than to those which qualify the rock to be a source (namely its high organic content), it is assumed that there is one rock formation with favorable impermeability and at the same time with favorable organic content. Note that the two attributes might be dependent, which means that the "weak link" approach explained by Rose (2001) may apply here. However, it would be misleading if the probability given to the occurrence of the formation were applied twice - first as a source rock component and then as a seal. 
The same conclusion can be drawn if the source and the reservoir are the same formation (f.i. Cretaceous limestone) or if a migration vehicle is also the reservoir (f.i. Pliocene sandstone).

\section{Conclusions and recommendations}

The above examples lead to the overall conclusion that special care is needed when the components of geological probability estimation are defined. I do not disagree that the industry standard guidelines do apply to the majority of the prospects. The five-component assessment methodology (Rose 2001), for instance, applies if the occurrences of source rock, reservoir, closure, migration and containment are the result of the favorable outcomes of independent geologic and hydrodynamic events. Once their independency is challenged, however, a slightly different approach is suggested, as follows:

Each prospect is described with a geologic model consisting of statements on the favorable outcome of the tectono-stratigraphic event framework, which leads to the conclusion that our prospect is in fact an accumulation with a recoverable quantity of petroleum. An example for such a statement might be: "During the Early Oligocene the 70-90 meters-thick marl formation was developed, which due to its high organic content and low permeability - can be considered as the source and seal rock of the prospect."

To begin with it is necessary to prepare the list of statements such as the one above. Note that special attention must be paid to the geologic and eventalgebraic independency of the events listed. As the second step of the assessment it is necessary to assume - based on the evaluation of the geologic and geophysical evidence - probability values for each statement. Finally, by means of multiplication of the probabilities of the favorable outcomes of all the indentified independent events, the Pg of the prospect is computed (see Equation 2).

The refined approach of geological probability estimation discussed in this paper may also open the way to the exploration success chance analysis of unconventional petroleum resources. At the same time it is assumed that customized implementation of the methodology in the geothermal energy exploration may bring results as well.

\section{References}

Howard, R.A. 1966: Decision Analysis: Applied Decision Theory. - In: Hertz D.B., J. Melese (Eds): Proceedings of the 4th International Conference on Operational Research, New York: WileyInterscience pp. 55-71.

Newendorp, P.D. 1975: Decision Analysis for Petroleum Exploration. - The Petroleum Publishing Co., Tulsa, OK.

Otis, R.M., N. Schneidermann 1997: A Process for Evaluating Exploration Prospects. - AAPG Bulletin, Vol. 81, No. 7.

Rose, P.R. 1992: Chance of Success and Its Use in Petroleum Exploration. - In: Steinmetz, R. (Ed.): The Business of Petroleum Exploration. AAPG Treatise of Petroleum Geology, Chapter 7, pp. 71-86.

Rose, P.R. 2001: Risk Analyses and Management of Petroleum Exploration Ventures. - In: AAPG Methods in Exploration Series No. 12. 